\title{
Understanding diffusion and density anomaly in a coarse-grained model for water confined between hydrophobic walls
}

\author{
Francisco de los Santos ${ }^{* \dagger}$ and Giancarlo Franzese $*, \hbar$ \\ Departamento de Electromagnetismo y Física de la Materia, Universidad de Granada, \\ Fuentenueva s/n, 18071 Granada, Spain, and Departamento de Física Fundamental, Universidad \\ de Barcelona, Diagonal 645, 08028 Barcelona, Spain \\ E-mail: fdlsant@ugr.es; gfranzese@ub.edu
}

\begin{abstract}
We study, by Monte Carlo simulations, a coarse-grained model of a water monolayer between hydrophobic walls at partial hydration, with a wall-to-wall distance of about $0.5 \mathrm{~nm}$. We analyze how the diffusion constant parallel to the walls, $D_{\|}$, changes and correlates to the phase diagram of the system. We find a locus of $D_{\|}$maxima and a locus of $D_{\|}$minima along isotherms, with lines of constant $D_{\|}$resembling the melting line of bulk water. The two loci of $D_{\|}$extrema envelope the line of temperatures of density maxima at constant $P$. We show how these loci are related to the anomalous volume behavior due to the hydrogen bonds. At much lower $T$, confined water becomes subdiffusive, and we discuss how this behavior is a consequence of the increased correlations among water molecules when the hydrogen bond network develops. Within the subdiffusive region, although translations are largely hampered,
\end{abstract}

\footnotetext{
*To whom correspondence should be addressed

${ }^{\dagger}$ Departamento de Electromagnetismo y Física de la Materia, Universidad de Granada, Fuentenueva s/n, 18071 Granada, Spain

†Departamento de Física Fundamental, Universidad de Barcelona, Diagonal 645, 08028 Barcelona, Spain
} 
we observe that the hydrogen bond network can equilibrate and its rearrangement is responsible for the appearance of density minima along isobars. We clarify that the minima are not necessarily related to the saturation of the hydrogen bond network.

\section{Introduction}

Water displays many thermodynamic and dynamic anomalies. $\underline{1}^{-3}$ Although the origin of these anomalies can usually be traced back to the properties of hydrogen bonding between water molecules, their quantitative understanding is a goal to which a great deal of effort is being devoted. Experiments show that these anomalies are evident where liquid water is stable, but are stronger below the melting line where water is supercooled, i.e. metastable with respect to crystal ice. Bulk water can be kept in this supercooled state down to about $235 \mathrm{~K}$ at $1 \mathrm{~atm} . \stackrel{4}{-}$ The experimental limit of stability of supercooled water with respect to crystal ice defines the homogeneous crystallization temperature $T_{h}(P)$, which reaches its lowest value of $181 \mathrm{~K}$ at about $2000 \mathrm{~atm}$. In an attempt to rationalize these anomalies, several ideas have been proposed, including the stability limit (SL) conjecture, $\frac{5}{}$ the liquid-liquid critical point (LLCP) scenario, $\underline{\underline{6}}$ the singularity-free (SF) hypotheses, $\underline{\underline{7}}$ and the critical point free $(\mathrm{CPF})$ scenario. $\underline{8}$ All these stimulating ideas are consistent with the experimental properties of water, but hypothesize different behaviors below $T_{h}(P)$. Although these differences cannot be directly tested in experiments, their implications in the interpretation of water properties could be different, or could be relevant for other anomalous liquids. $\stackrel{9-11}{-1}$ It is therefore worthwhile to test these hypothesis in theoretical models. Many authors resort to computer simulations of detailed models of water (see for example ${ }^{12}$ for a short updated list). However, this approach, both

for molecular dynamics (MD) 13 or Monte Carlo (MC) simulations, 14 faces the problem of large computational times near $T_{h}(P)$, because water equilibration time increases in an exponential way for decreasing $T$.

An alternative approach, which we follow here, is to consider a coarse-grained model of water that allows to perform, on the one hand, efficient MC simulations and, on the other hand, theo- 
retical calculations. In particular, we focus on a model for a water monolayer confined between hydrophobic walls. $\stackrel{15,16}{1}$ The interest for this case comes from the fact that, under appropriate conditions, the formation of ice can be avoided in experiments with water under confinement. $\underline{\underline{17}}-\underline{19} \mathrm{We}$ consider here only the case of confinement between infinite hydrophobic walls, while other kind of confinements, mimicking porous hydrophobic materials, have been considered in other works. $\underline{\underline{20}}$

The coarse-grained model considered here allows to gain an insight into the properties of the scenarios that have been proposed for supercooled water. In particular, it is possible to show that within the framework of this model all the scenarios proposed for supercooled water differ only in the relative strength of the directional (covalent) and the many-body (cooperative) component of the hydrogen bond (HB). $\cdot \underline{21}$ When the many-body HB component is strong and the directional HB component is weak, the model recovers the CPF scenario, and shows that it coincides with the SL case. When the many-body HB component is zero, the model reproduces the SF scenario for any finite directional HB component. Finally, for intermediate values of the two HB components, the model recovers the LLCP scenario, with the liquid-liquid phase transition having a negative slope in the pressure-temperature $(P-T)$ phase diagram. The LLCP occurs at positive or negative pressure depending of the relative strength of the many-body HB component with respect to the directional HB component. $\underline{21}$ Direct experimental evaluation of the relative strengths of the two HB components is not straightforward, but indirect evaluations are consistent with values that, for the model, would predict the LLCP scenario. $\underline{\underline{21}}$

\section{Dynamic properties}

Among the many dynamics anomalies of water, we will focus here on the behavior of translational (self)diffusion constant $D$. In normal liquids $D$ decreases when $P$ increases at constant $T$, while water displays a large increase of $D$ in a delimited region of $P-T, \underline{22,23}$ e. g., with an enhancement of about $60 \%$ at $243 \mathrm{~K}$ when $P$ increases from $0.1 \mathrm{MPa}$ to $150 \mathrm{MPa} \cdot \underline{\underline{23}}$ The increase is observed up to about $200 \mathrm{MPa} \cdot \underline{.24,25}$

Computer simulations of bulk water with detailed models (see Ref.s $\underline{26} \underline{\underline{30}}$ and references therein) 
and lattice models (e. g..$^{31-33}$ ) can reproduce, at least qualitatively, the anomalous behavior of $D$. By analyzing the microscopic structure of water molecules in the region of the anomalous increase of $D$, several authors have proposed a relation between the behavior of $D$ and the structure of water. For example, Ref. $\underline{34}$ relates $D$ to the configurational entropy, Ref. $\underline{35}$ associates the minima in $D$ with a maximum in orientational order, and Ref. $\underline{36}$ shows, for a water-like isotropic potential, the connection of the anomaly in $D$, and in other quantities, with the density dependence of the entropy in excess over the entropy of the ideal gas. In particular, by classical molecular dynamic simulations it has been observed that the increase of $P$ weakens the hydrogen bonds, and thus increases $D . \stackrel{28,29}{2}$ This interpretation in terms of defects in the HB network can be extended to negative $P . \underline{30}$ A similar qualitative conclusion has been reached also by ab initio molecular dynamics showing that $D$ is directly linked to network imperfections. ${ }^{37}$ Nevertheless, a quantitative relation between the anomalous behavior of $D$ and the microscopic structure of water is still missing.

In confinement, experiments show controversial results. Without the aim of reviewing the relevant literature, we recall here, as examples, that the reduction of water $D$ can be of up to two orders of magnitude between $260 \mathrm{~K}$ and $310 \mathrm{~K}$, in hydrophilic $\mathrm{NaX}$ and $\mathrm{NaA}$ zeolites, $\underline{38}$ or that the viscosity of water between two hydrophilic surfaces, with $\leq 1 \mathrm{~nm}$ interfacial separation, is seven orders of magnitude greater than that of bulk water at room temperature. $\frac{39}{}$ Other experiments display that $D$ decreases if the confinement increases, e. g. in MCM-41 with pore radius between $1 \mathrm{~nm}$ and $2 \mathrm{~nm} \stackrel{40-43}{-43}$ or MCM-48 with pore radius of about $1 \mathrm{~nm}, \underline{41}$ both slightly hydrophilic due to the oxygen atoms in the silica structure, with the first considered more hydrophobic than the second. Similar results were observed for channels of closed multiwalled hydrophobic carbon nanotubes with diameter between $2 \mathrm{~nm}$ and $5 \mathrm{~nm} . \underline{44}$ Nevertheless, other experiments reveal an exceptionally fast mass transport for water confined in carbon nanotubes of about $2 \mathrm{~nm}^{45}$ and $7 \mathrm{~nm}$ radius. $\underline{46}$

Computer simulations have been performed to rationalize the different experiments. By MD it has been found a decrease of $D$ with decrease of separation between two hydrophilic surfaces at nanoscopic distance for SPC/E water. $\stackrel{47}{ }$ Moreover, it has been shown for the same model that the decrease of hydration largely decreases the mobility of water molecules near the surface of 
MCM-41 or Vycor, consistent with the interpretation that at low hydration the majority of them are bonded to the surface. $\underline{48}$

In the case of hydrophobic confinement, the results are more controversial. MD of TIP5P water nanoconfined between hydrophobic smooth walls displays anomalous $D, \underline{49}$ but only in the direction parallel to the walls. ${ }^{50} \mathrm{D}$ results to be two orders of magnitude smaller than in bulk and with the anomaly occurring in confinement at lower $T$ than in bulk. $\underline{49}$ A similar large decrease of mobility has been reported at ambient conditions for SPC/E water between two large hydrophobic graphite-like plates for separations below $1.3 \mathrm{~nm} . \underline{51}$ Nevertheless, first-principle MD simulations of the same model in similar conditions show that the diffusion of water molecules become faster under confinement, possibly due to weaker HBs at the interface. $\stackrel{52}{2}$ A similar controversy is reported for simulations of water in carbon nanotubes, with radii below $1 \mathrm{~nm} . \underline{52}-\underline{55}$

\section{Our approach}

Our approach to the problem is to study by MC the local dynamics of a coarse-grained model of confined water that will be defined in detail in the following. The results described in the previous section for this model have been derived by free-energy calculations within mean-field approach and efficient MC simulations. In particular, a cluster MC dynamics allows to easily equilibrate the model at any $T$ and $P . \underline{56}$ This specific MC approach is based on a mapping of the thermodynamic model into a geometrical problem, using an appropriate extension of the correlated-percolation approach. $57-60$ Nevertheless, as an alternative it is possible to adopt a local MC algorithm with the aim of studying the dynamic behavior of the model and compare it with the experimental behavior of water. $\underline{61,62}$

This kind of study is useful for systems at equilibrium, although approaching a glassy dynamics, $\underline{59,63,64}$ and makes the plausible assumption that, at a given $T$ and $P$, the MC time step can be converted into real time units by a factor that does not depend on time. This assumption is consistent with the mode-coupling theory (MCT), according to which the long-time relaxation $(\alpha-$ relaxation) dynamics should be independent of the microscopic dynamics, $\underline{65}$ as tested in Lennard- 
Jones mixtures. $\frac{66}{}$ However, the comparison must be performed with caution, because the time conversion factor for a given observable could depend on both $T$ and $P$, when the interactions are non-isotropic. ${ }^{67}$ This dependence can be estimated by comparing MC results with experiments, as done by Mazza et al. in Ref. $\underline{68}$ for the model under consideration here. The comparison shows a linear relation between the logarithms of time in real units and time in MC step at different $T$ and constant $P, \underline{68}$ corresponding to a power-law relation between the two times. This relation can be easily understood in the context of the present coarse-grained model, at least at low $T$. At low enough $T$, both the experimental time and the MC time follows a generalized Arrhenius relation 68 where the characteristic $T$-dependent activation energy corresponds to the energy needed to break a HB. The power-law relation between the two times turns out to be a consequence of the choice of the model parameters that implies a HB energy lower than in experiments. Therefore, by choosing appropriate model parameters the two times would be directly proportional, with a proportionality factor that would not depend on $T$ at constant $P$.

With this caveat in mind, we perform here an extensive MC study of the thermodynamic and dynamic behavior of the coarse-grained model, presented in Ref., $\underline{15}, 16$ for a water monolayer confined between hydrophobic walls. In particular, we consider the case in which water molecules can diffuse. We identify the region where diffusion has an anomaly behavior, finding maxima and minima of the diffusion coefficient at fixed $T$, and we discuss how this anomaly is related to other anomalies of water.

In particular, thanks to the feature that the model can be tuned from one scenario to another, 21 we can test if the diffusion anomaly is related to some specific scenario for the thermodynamics of the system at much lower $T$. Specifically, we consider the LLCP and the SF scenarios. We do not observe any relevant differences in the region where the system displays diffusion anomaly.

We finally investigate the very low $T$ dynamics, observing a subdiffusive regime and the approach to a glassy state at any $P$. Under these conditions, we observe density minima, resembling recent experiments $\frac{69}{}$ and MD simulations..$^{70,71}$ In the present model, the density minima appear in the vicinity of the glassy state and as a consequence of the breaking of HBs to rearrange water 
molecules for a better matching with the local order. In particular, we observe that if the model does not take into account the many-body interaction, the density minima are largely reduced.

\section{Coarse-grained model of a water monolayer}

We consider a water monolayer confined between two hydrophobic smooth walls with double periodic boundary conditions. The walls are separated by a distance of about $h=0.5 \mathrm{~nm}$. The system is considered at constant number $N$ of water molecules, constant $T$ and constant $P$, leaving the volume $V$ free to change. For a TIP5P-water monolayer confined between hydrophobic walls it has been observed that, depending on the separation $h$, water is liquid or forms a quasi-twodimensional square ice for temperatures ranging between $260 \mathrm{~K}$ and $300 \mathrm{~K}$, and negative lateral pressure. $\frac{49,72}{}$ The square symmetry is a consequence of the distortion imposed by the walls to the tetrahedral HB network that would otherwise form in bulk water. Consistently with these findings, we divide the available volume $V$ into $\mathscr{N}$ square cells, each with volume $v=V / \mathscr{N}$, square section of size $\sqrt{v / h}$ and height $h$, and hydrate the system with $N \leq \mathscr{N}$ water molecules.

In our coarse-graining we assume that each cell can at most host one water molecule. Therefore, if $N=\mathscr{N}$, then each cell has one molecule and the system is homogeneous in density. If $N<\mathscr{N}$, some cells are empty. To each cell we associate an occupation variable $n_{i}=0,1$ $(i=1,2, \ldots \mathscr{N})$ if it is vacant or occupied, respectively. Having only one water molecule per cell between the walls, for the sake of simplicity, we reduce the description of the monolayer to a two-dimensional system.

The water-water interaction is decomposed into three terms. The first accounts for all the isotropic contributions, including short-range electronic orbitals repulsion and van der Waals attraction, and is represented by a Lennard-Jones potential truncated at a hard-core diameter $r_{0}$

$$
U(r) \equiv \begin{cases}\infty & \text { for } r \leq r_{0} \\ \varepsilon\left[\left(\frac{r_{0}}{r}\right)^{12}-\left(\frac{r_{0}}{r}\right)^{6}\right] & \text { for } r>r_{0}\end{cases}
$$


where $\varepsilon$ is the interaction energy and $r$ is the distance between two molecules. In the coarse-grained representation, $r$ is the distance between the center of occupied cells. The hard-core diameter $r_{0}$ is introduced to simplify the implementation of the model and our tests show that the results do not depend on its existence. We set $r_{0} \equiv 2.9 \AA$, the water van der Waals diameter, $\stackrel{73,74}{ }$ and $\varepsilon \equiv 5.8 \mathrm{~kJ} / \mathrm{mol}$, consistent with the value $5.5 \mathrm{~kJ} / \mathrm{mol}$ of the estimate of the van der Waals attraction based on isoelectronic molecules at optimal separation. $\underline{75}$

The second term of water-water interaction accounts for the directional (covalent) component of the HB formation, $\underline{76,77}$ with a characteristic energy that we set $J \equiv 2.9 \mathrm{~kJ} / \mathrm{mol}$. To account for the directionality we adopt a geometrical definition in which the $\mathrm{HB}$ breaks if $\widehat{\mathrm{OOH}}>30^{\circ}$. Therefore, only $1 / 6$ of the orientation range $\left[0,360^{\circ}\right]$ in the $\mathrm{OH}-\mathrm{O}$ plane is associated with a bonded state. We therefore associate to each molecule $i$ a bonding index $\sigma_{i j} \in[1,2, \ldots q]$ with a discrete number of states $q$ describing the bonding state with a neighbor molecule $j$, and choose $q \equiv 6$ to account for the entropy loss associated with the formation of a HB. Due to the square symmetry, each molecule has four neighbors and four bonding indices $\sigma_{i j}$. Therefore, each molecule has $q^{4}=6^{4}=1296$ possible bonding states. To form a HB between two molecules in two occupied neighboring cells $i$ and $j$ (hence $n_{i} n_{j}=1$ ) we assume that the two facing bonding indices $\sigma_{i j}$ and $\sigma_{j i}$ are in the same state, i.e. $\delta_{\sigma_{i j}, \sigma_{j i}}=1$, with $\delta_{a, b} \equiv 1$ if $a=b$, and $\delta_{a, b} \equiv 0$ otherwise. Therefore, the directional term of the HB can be expressed as

$$
H_{J} \equiv-J N_{H B},
$$

where

$$
N_{H B} \equiv \sum_{\langle i, j\rangle} n_{i} n_{j} \delta_{\sigma_{i j}, \sigma_{j i}}
$$

is the number of HBs and the sum runs over all nearest-neighbor cells.

The directional interaction of HBs leads to local reduction of density as a consequence of the reduced nearest neighbors with respect to close molecular packing. This property is at the origin of the macroscopic density maximum that occurs at $4^{\circ} \mathrm{C}$ at ambient pressure, a temperature below which the number of HBs per molecule is about $3 . \underline{78}$ The effect can be observed in experiments 
mainly as a change in the structure of the second shell of water molecules. $\stackrel{74,79-81}{-1}$ Nevertheless, to include the effect in a tractable way in the coarse-grained model, we follow Ref. $\underline{\underline{7}}$ and consider that each formed HB leads to a small increase of volume $v_{H B}$ per molecule, where $v_{H B} / v_{0} \equiv 0.5$ is the average density increase from low density ice Ih to high density ices VI and VIII and $v_{0} \equiv h r_{0}^{2}$ is our approximation to the van der Waals volume of a molecule. Therefore, the total volume occupied by water is

$$
V_{w} \equiv N v+N_{H B} v_{H B}
$$

Note that the increase $v_{H B}$ corresponds to a larger volume per molecule, but not to a larger separation $r$ between molecules, hence it does not affect the radial term in ??.

The last term we include in the water interaction energy is a many-body (cooperative) interaction among $\mathrm{HBs}$, that favors specific values of the probability distribution of $\mathrm{O}-\mathrm{O}-\mathrm{O}$ angles $\underline{80,81}$ (see also Ref. ${ }^{25}$ for a brief description of the quantum origin of the cooperative interaction). Furthermore, the probability distribution of $\mathrm{O}-\mathrm{O}-\mathrm{O}$ changes when comparing bulk and confined water, ${ }^{82}$ showing the disappearing of the fifth interstitial neighbor in the confined case, and a shift of the maximum of the distribution toward $90^{\circ}$ at low $T$, consistent with the symmetry chosen for the coarse-graining in our model. To account for this cooperative interaction, we include in the model the term

$$
H_{\text {Coop }}=-J_{\sigma} \sum_{i} n_{i} \sum_{(k, l)_{i}} \delta_{\sigma_{i k}, \sigma_{i l}}
$$

where $(k, l)_{i}$ stands for the six different pairs of four bonding indices of a molecule $i$, and $J_{\sigma}$ is related to the energy gain when the bonding indices order in the same state, with the maximum gain $-6 J_{\sigma}$ per molecule, corresponding to the fully ordered case.

As shown by Stokely et al., $\frac{21}{}$ by setting the parameters $\varepsilon, J$ and $v_{H B}$ to finite values and tuning the parameter $J_{\sigma}$, it is possible to reproduce all the scenarios that have been proposed for supercooled water. Here we consider two cases: (i) $J_{\sigma}=0.29 \mathrm{~kJ} / \mathrm{mol}$, corresponding to the LLCP scenario, and (ii) $J_{\sigma}=0$, corresponding to the SF scenario.

The choice in (i) is consistent with the experimental measure of HBs in ice Ih, approximately 
$3 \mathrm{~kJ} / \mathrm{mol}$ stronger than in liquid water. $\underline{83}$ If we entirely attribute this increase to the cooperative component of the $\mathrm{HB}, \underline{84}$ we find $J_{\sigma} \simeq 0.25 \mathrm{~kJ} / \mathrm{mol}$ for the two molecules forming the $\mathrm{HB}$ (each with an energy gain in absolute value $6 J_{\sigma}$ ), which is consistent with our choice.

The water-wall interaction is represented by a hard-core exclusion. Although the interaction of water with a hydrophobic, infinitely large, object could have a small attractive van der Waals component or a soft repulsion, for the sake of simplicity we assume here that the main effect of the confining walls is to inhibit the formation of ice, as observed by Zangi and Mark for $h<0.51$ $\mathrm{nm} . \underline{72}$

Therefore, the enthalpy of the system at pressure $P$ is

$$
H \equiv U(r)+H_{J}+H_{\text {Coop }}+P V_{w} .
$$

For a given occupancy ratio $N / \mathscr{N}$, the state of the system is fully specified by the average number density $N / V$ and the set of $\sigma_{i j}$.

\section{The Monte Carlo method}

We perform MC simulations in the $N P T$ ensemble for a system partitioned into $\mathscr{N}=2500$ square cells and an occupancy ratio $N / \mathscr{N}=0.75 \%$, corresponding to $N=1875$ water molecules. Since we allow for changes of the volume in the direction parallel to the walls, the control parameter $P$ represents the pressure parallel to the walls. To test for size effects, we consider also $\mathscr{N}=400$ and $\mathscr{N}=1600$ at the same $75 \%$ occupancy ratio, with $N=300$ and $N=1200$ respectively. Our results do not show any appreciable size effect among these three cases. Likewise, we do not find any significant differences for occupancy ratios between $75 \%$ and $90 \%$.

MC and mean-field results for this model at full occupancy $15,61,62,85-92$ were reported in previous works. $\underline{16,20,21,56,60,68,93-95}$ An analysis of the differences between partial and full occupancy will be presented elsewhere.

To generate equilibrium configurations we pick at random a cell $i \in\{1, \ldots, N\}$ and an integer 
$j \in\{0,1,2,3,4\}$. If $j=0$, we choose at random one of the four neighboring cells of the cell $i$ and, if empty, we displace into it the molecule $i$ with probability given by the following ?? of a Metropolis algorithm.

If $j \geq 1$ we set the bonding index $\sigma_{i j}$ to any of the $q$ possible states, independent of its original state, and accept the change with probability in ??. One MC step consists of $5 N$ of these trials followed by a volume-change attempt, in which we select a new volume at random in the interval $[V-\delta V, V+\delta V]$ with $\delta V=0.5 v_{0}$, and accept the move with probability in ??. Since we change the volume $V$ in a continuous way, the volume per cell $v$ and the distance between the center of cells $r$ also change in a continuous way, as in an off-lattice model, despite the fact that the model has a fixed maximum number of nearest neighbors, equal to four, due to the square lattice symmetry adopted in the coarse-graining.

From the new and old configurations we calculate $\Delta H \equiv H^{\text {new }}-H^{\text {old }}$ from ??, and $\Delta S \equiv$ $-N k_{B} \ln \left(V^{\text {new }} / V^{\text {old }}\right)$, where $k_{B}$ is the Boltzmann constant. We accept the new configuration with probability

$$
\min \{1, \exp [-\beta(\Delta \mathrm{H}-\mathrm{T} \Delta \mathrm{S})]\}
$$

where $\beta=1 /\left(k_{B} T\right)$.

Following Ref. $\underline{68}$ we adopt real units to represent our results. The transformations to real units are based on rescaling the MC $T, P$, and $t$ from experimental data for a monolayer of water adsorbed on lysozyme powder $\frac{68}{}$ and adjusting the $\mathrm{MC} \rho$ to experimental values around the maximum density at ambient pressure in a self-consistent way. These transformations are meant to give the order of magnitude of the calculated quantities.

In the calculation of the Lennard-Jones interaction energy in ?? we test that there is no appreciable difference if we introduce a cut-off at the 9th neighbor for the maximum interaction range. To allow for a better equilibration of the system, we follow an annealing protocol along isobars, starting at high $T$, with $\mathscr{N}$ cells randomly occupied by $N$ water molecules, each molecule having a random configuration of bonding indices, and with the total volume $V=\mathscr{N} v_{0}$. We equilibrate each state point for $0.2 \mathrm{~ms}$ and produce data for $15 \mathrm{~ms}$. 
We calculate the coefficient $D_{\|}$of water diffusion parallel to the plates from the Einstein relation in two dimensions

$$
D_{\|} \equiv \lim _{t \rightarrow \infty} \frac{\left\langle\left|\mathbf{r}_{i}\left(t+t_{0}\right)-\mathbf{r}_{i}(t)\right|^{2}\right\rangle}{4 t}
$$

where $\mathbf{r}_{i}(t)$ denotes the projection onto the plates of the position of molecule $i$ at time $t$, and $\langle\cdot\rangle$ stands for the average over all molecules $i$ and over different values of $t_{0}$.

To avoid correlations in the calculations of $D_{\|}$and all the other quantities, we perform averages over blocks of $\tau_{0}=0.8 \mu \mathrm{s}$, sampled every $80 \mathrm{ps}$. We check that $\tau_{0}$ approximately corresponds to the time needed for a molecule to reach its image points for most of the temperatures investigated. However, for the lowest temperatures, we use up to $10 \tau_{0}$ as block-length for averages.

\section{Results and discussion}

\section{Phase diagram}

At high $T$ we find the gas phase, separated from the liquid phase by a first-order phase transition ending in a critical point ([figure $][1][] 1)$. The liquid-gas critical point for the hydrophobically confined monolayer occurs at a pressure and temperature that are higher than that of bulk water, qualitatively consistent with the results of MD simulations for TIP4P water in hydrophobic confinement. $\underline{96}$

By annealing the system from high temperatures, we find a discontinuous change in density

along isobars $\left(\left[\right.\right.$ figure][2][]2). This change occurs at the spinodal temperature $T_{S}^{\mathrm{LG}}(P)$, that marks the limit of stability of the gas phase with respect to the liquid phase. The change is very large at low $P$ and vanishes as the liquid-gas critical point is approached. At the critical point, by definition, the isobar has infinite negative slope. At $P$ higher than the critical pressure, the minimum slope of $\rho(T)$ become finite and decreases in absolute value for increasing $P$. The locus of these minima corresponds to the locus of maxima of the isobaric thermal expansion coefficient $\alpha_{P}^{\max }(P) \equiv \min _{\mathrm{T}}\left\{-\partial \ln \left(\rho \mathrm{v}_{0}\right) /\left.\partial \mathrm{T}\right|_{\mathrm{P}}\right\}$, with the maxima decreasing in value by increasing $P$. 


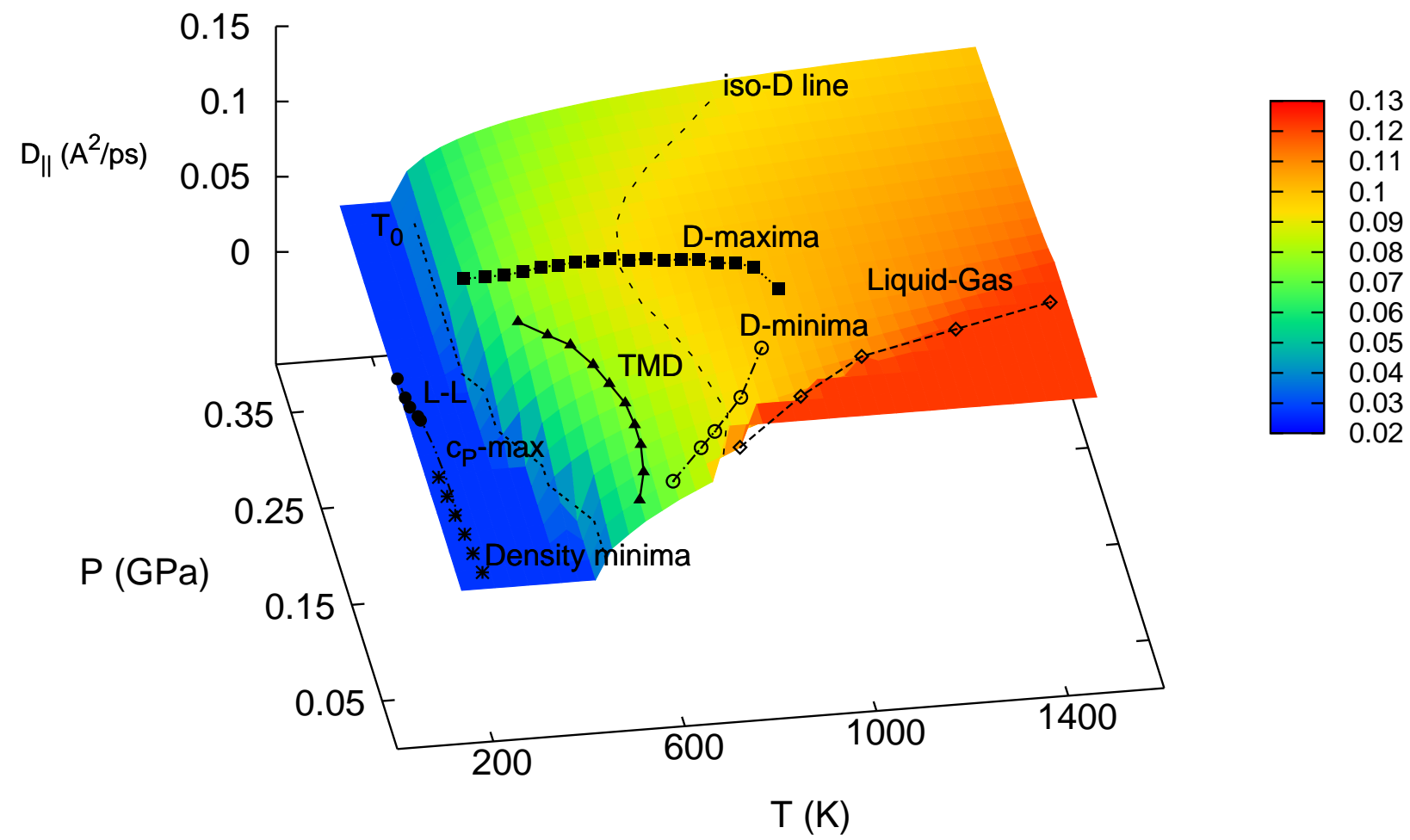

Figure 1: $P-T$ phase diagram of a water monolayer nanoconfined between hydrophobic plates in which we emphasize how the diffusion constant $D_{\|}(z$-axis and color scale) changes in relation with the thermodynamic behavior. The liquid-gas phase transition line (open diamonds) ends in the liquid-gas critical point (diamond symbol at highest $T$ ). The loci of isothermal maxima of $D_{\|}$, $D_{\|}^{\mathrm{MAX}}$ (solid squares), and minima, $D_{\|}^{\min }$ (open circles), include the TMD line (solid triangles). Loci at constant $D_{\|}$(e.g. the dashed line marked as 'Iso- $D$ ') resemble in their reentrant behavior the water melting line. $D_{\|}$values above $0.12 \AA / \mathrm{ps}^{2}$ (gas phase) and below $0.03 \AA / \mathrm{ps}^{2}$ are not color-graded. State points with $D_{\|}<0.03 \AA / \mathrm{ps}^{2}$ are below the onset $T_{o}(P)$ (dotted line) of the subdiffusive regime and their diffusion coefficient cannot be defined. For $T<T_{o}(P)$, we observe a locus of density minima (asterisks), a locus of discontinuity in density (solid circles), a locus of maxima of $C_{P}$ (dot-dashed line) as in a liquid-liquid phase transition ending in a critical point. Here we use $\varepsilon=5.8 \mathrm{~kJ} / \mathrm{mol}, J=2.9 \mathrm{~kJ} / \mathrm{mol}, J_{\sigma}=0.29 \mathrm{~kJ} / \mathrm{mol}, v_{H B} / v_{0}=0.5$ as parameters for the coarse-grained water model.

By changing the simulation protocol across $T_{S}^{\mathrm{LG}}(P)$, i.e. heating the system instead of annealing it, we find the typical hysteresis associated to a first-order phase transition, with the hysteresis vanishing as the liquid-gas critical point is approached. All these results are consistent with 

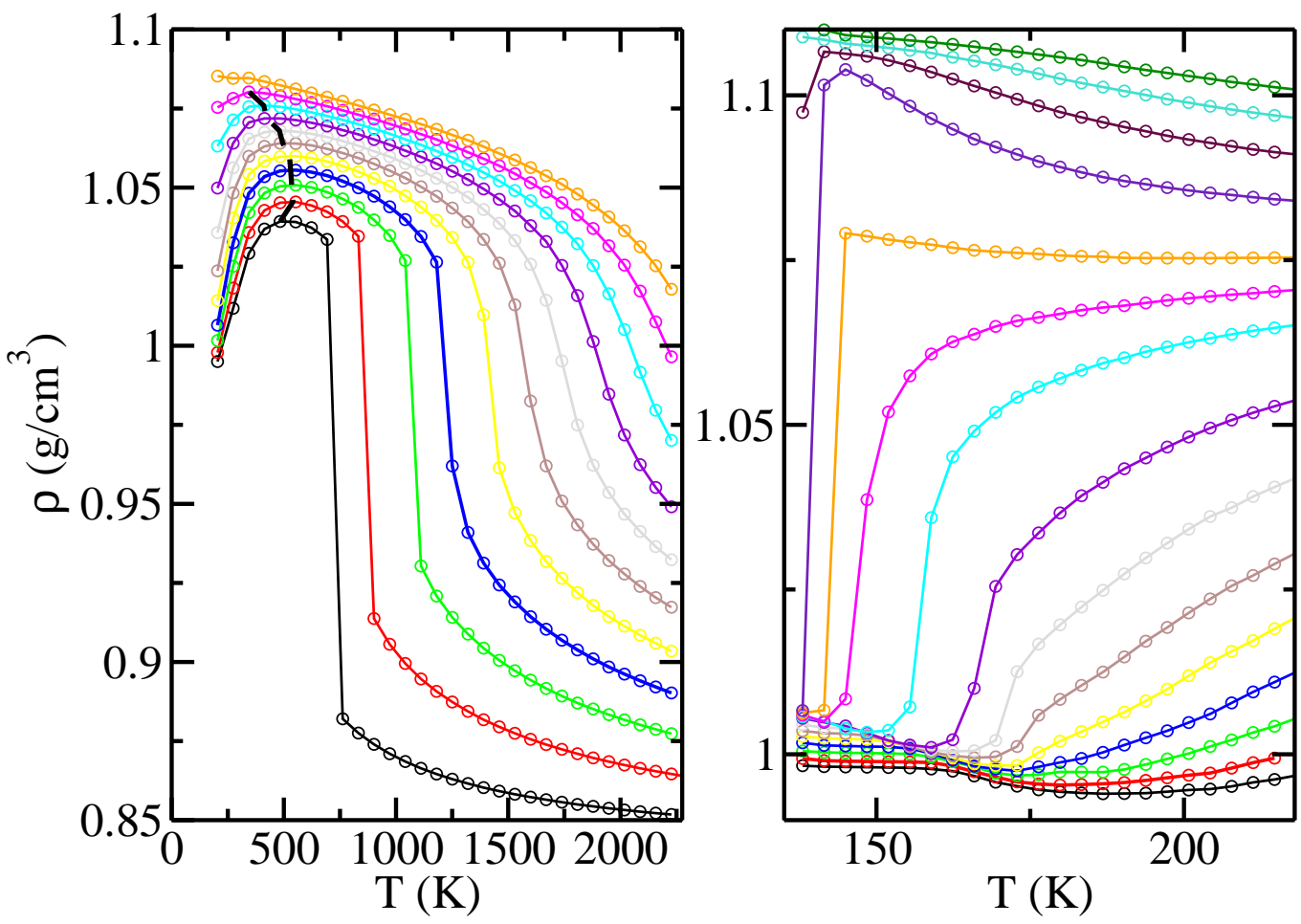

Figure 2: Density $\rho$ as a function of temperature $T$ along isobars at $0.02 \mathrm{GPa} \leq P \leq 0.22 \mathrm{GPa}$ separated by $0.02 \mathrm{GPa}$ increments, as calculated by $\mathrm{MC}$ annealing. (Left panel) At low $P$, the discontinuity in $\rho$ marks the gas-to-liquid spinodal temperature $T_{S}^{\mathrm{LG}}(P)$, vanishing at higher $P$ into the liquid-gas critical point. The dashed line marks the temperature $T_{\mathrm{MD}}(P)$ of maximum density. For clarity we show only a selection of the simulated state points. (Right panel) Enlarged view at low $T$ along isobars at $0.02 \mathrm{GPa} \leq P \leq 0.30 \mathrm{GPa}$ separated by $0.02 \mathrm{GPa}$ increments. Model parameters as in [figure][1][]1. 
previous findings for this model. $15,16,20,21,56,60-62,68,85-95$ Here we add the information about the diffusion constant.

\section{Diffusion maxima and minima}

Upon crossing the liquid-gas phase transition, the diffusion constant $D_{\|}$displays a discontinuous change that vanishes as the critical point is approached. Above the critical point, $D_{\|}$changes in a continuous way, as expected in a one-phase region.

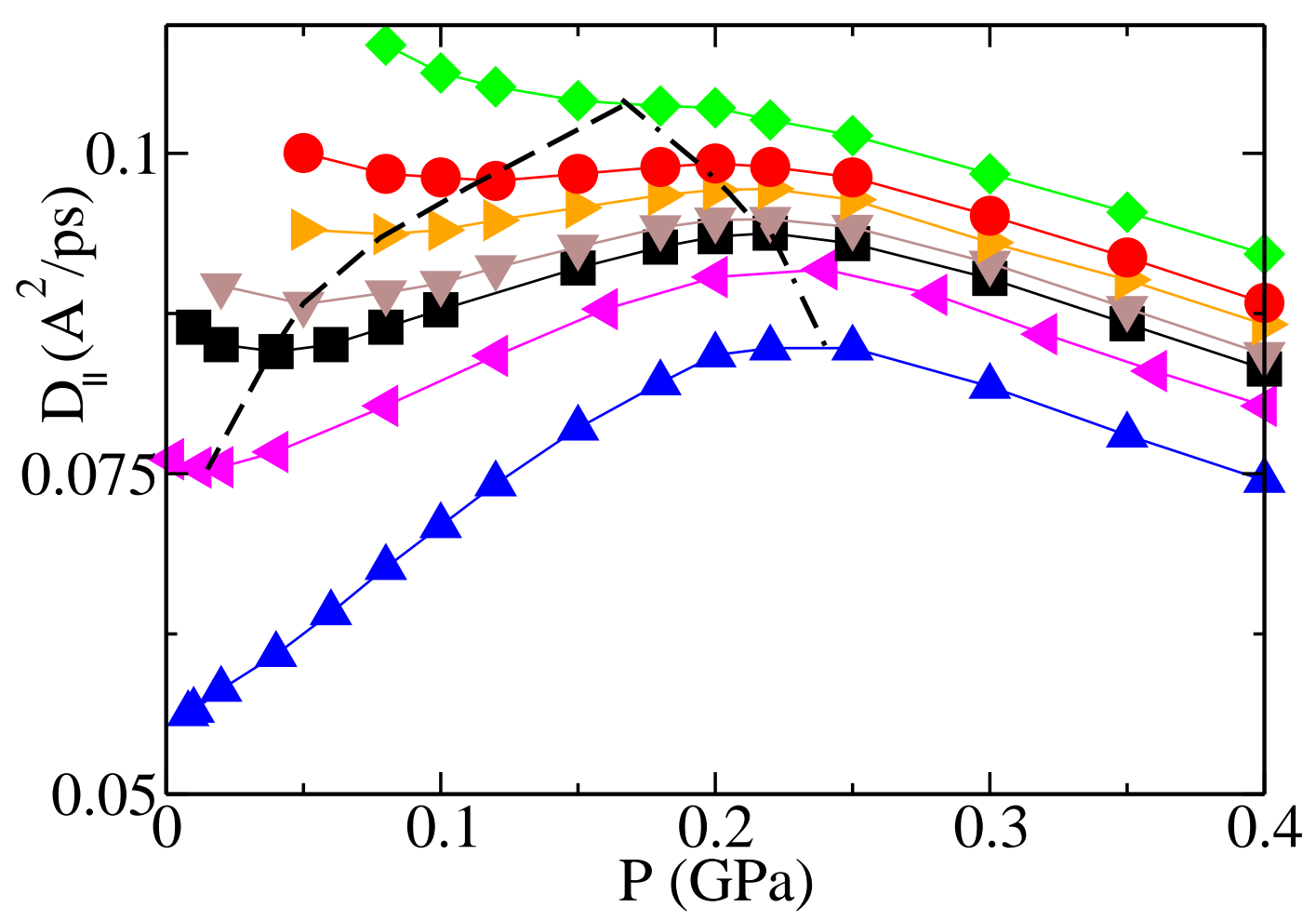

Figure 3: Diffusion coefficient $D_{\|}$as a function of pressure along isotherms for (from bottom to top) $T=483 \mathrm{~K}, 588 \mathrm{~K}, 658 \mathrm{~K}, 693 \mathrm{~K}, 762 \mathrm{~K}, 832 \mathrm{~K}$ and $1006 \mathrm{~K}$. For $T>832 \mathrm{~K}, D_{\|}$is monotonic with $P$, while it has maxima $D_{\|}^{\mathrm{MAX}}$ and minima $D_{\|}^{\min }$ for $588 \mathrm{~K} \leq T \leq 832 \mathrm{~K}$, and a maximum for $T=483 \mathrm{~K}$. The pressure $P^{\mathrm{D}-\mathrm{MAX}}$ for $D_{\|}^{\mathrm{MAX}}$ (dot-dashed line) decreases for increasing $T$ and converges to the pressure $P^{\mathrm{D}-\text { min }}$ for $D_{\|}^{\min }$ (dashed line). $P^{\mathrm{D}-\text { min }}$ decreases for decreasing $T$ and, possibly, becomes negative. Model parameters as in [figure][1][]1 
In the liquid phase for $T>832 \mathrm{~K}, D_{\|}$decreases monotonically for increasing $P$, but has an anomalous non-monotonic behavior at lower $T$ ([figure][3][]3). This behavior resembles the known diffusion anomaly of bulk water. In particular, we find that $D_{\|}$decreases below a maximum $D_{\|}^{\mathrm{MAX}}$ for decreasing pressure at $P \lesssim 0.2 \mathrm{GPa}$, as observed in bulk water. $\underline{24.25}$ The pressure at which we find $D_{\|}^{\mathrm{MAX}}, P^{\mathrm{D}-\mathrm{MAX}}$, increases with decreasing $T$. At lower $P, D_{\|}$reaches a minimum $D_{\|}^{\min }$ at a pressure $P^{\mathrm{D}-\mathrm{min}}$ that decreases with decreasing $T$ and eventually becomes negative for $T<588 \mathrm{~K}$ ([figure][1][]1).

As a consequence of the occurrence of $D_{\|}^{\mathrm{MAX}}$ and $D_{\|}^{\min }$, the state points with the same value of $D_{\|}$forms lines in the $P-T$ phase diagram that are not monotonic as a function of $P$. Therefore, these lines at constant $D_{\|}$, or iso- $D_{\|}$lines ([figure][1][]1), have a positive slope in $P-T$ phase diagram for $P>P^{\mathrm{D}-\mathrm{MAX}}$ and for $P<P^{\mathrm{D}-\mathrm{min}}$, but a negative slope for $P^{\mathrm{D}-\mathrm{MAX}}>P>P^{\mathrm{D}-\mathrm{min}}$. It is interesting to observe that this change of slopes in $P-T$ plane resembles the change of slope of the bulk water melting line, at least for $P>P^{\mathrm{D}-\text { min }}$. Although the present model does not include any representation for the crystal, because we make the hypothesis that crystallization is avoided under the conditions considered here, our finding of the change of slope of the iso- $D_{\|}$lines suggests that the shape of the melting line in nanoconfinement could resemble that of bulk water. Moreover, our observation hints that its shape would be mainly determined by the slowing down of the dynamics.

Finally we observe that the temperatures of $D_{\|}^{\mathrm{MAX}}$ and $D_{\|}^{\min }$ are higher than those found in confinement and bulk with MD simulations of TIP5P-water. $\underline{49}$ This difference could be consistent with the higher values observed here for the liquid-gas spinodal line. On the other hand, the pressure at which we observe the onset of $D_{\|}^{\mathrm{MAX}}$ is consistent with experimental results for bulk water. $\underline{24.25}$

\section{Density maxima and expansivity minima}

By decreasing $T$ along isobars, we find maxima in density at a temperature $T_{\mathrm{MD}}(P)$ for $P \lesssim$ $0.2 \mathrm{GPa}$, as observed in bulk water 24,25 ([figure][2][12). Above $P \simeq 0.2 \mathrm{GPa}$ the density increases regularly for decreasing $T$, while at lower $P$ and moderate temperature $T<T_{\mathrm{MD}}(P)$, the density 
of liquid water decreases for decreasing $T$. As a result of this anomalous density behavior the isobaric thermal expansion coefficient (or expansivity) $\alpha_{P}(T) \equiv-\partial \ln \left(\rho v_{0}\right) /\left.\partial T\right|_{P}$ becomes negative for $T<T_{\mathrm{MD}}(P)$, as observed in bulk water. $\frac{1}{}$

The temperature $T_{\mathrm{MD}}(P)$ of maximum density has, in the $P-T$ phase diagram, a shape that resembles the one observed in bulk and in nanoconfined water, $\stackrel{49}{ }$ reaching a maximum temperature at about $50 \mathrm{MPa}$, similar to what is found in the TIP5P water model. $\underline{49}$ As expected for water, $\underline{35}$ we find the $T_{\mathrm{MD}}$ locus within the region where the diffusion anomaly occurs, delimited between $P^{\mathrm{D}-\mathrm{MAX}}(T)$ and $P^{\mathrm{D}-\min }(T)$ [figure][1][]1].

For $T<T_{\mathrm{MD}}(P)$ we find that the slope of $\rho(T)$ along isobars increases by decreasing $T$ ([figure][2][]2), implying a more pronounced negative value for $\alpha_{P}$, consistent with experiments for bulk $\underline{97}$ and supercooled confined water. $\underline{69.98}$ The slope decreases at lower $T$, consistent with the occurrence of a minima in $\alpha_{P}$, expected below $240 \mathrm{~K}$ from experiments in bulk water at ambient pressure. $\frac{99}{}$

\section{Subdiffusion}

Before describing in detail our findings about the density at lower $T$, we observe that for $T \leq 275 \mathrm{~K}$ at low $P=0.02 \mathrm{GPa}$, within our simulation time $\simeq 0.01 \mathrm{~s}$, the system does not reach the diffusive regime, i.e. the mean square displacement $\left\langle\left|\mathbf{r}_{i}\left(t+t_{0}\right)-\mathbf{r}_{i}(t)\right|^{2}\right\rangle$ is no longer proportional to the time $t\left[[\right.$ figure $][4][14)$. We find that the long-time behavior $\left(t>10^{6} \mathrm{ps}\right)$ is well described by the subdiffusive relation $\Delta r^{2} \sim t^{\alpha}$, with $\alpha=0.7$.

Subdiffusive behavior is observed in experiments for water hydrating mygloblin, at a hydration level corresponding to approximately one water monolayer. $\underline{100}$ The experiments show water subdiffusion at 320 and $300 \mathrm{~K}$, with an exponent $\alpha=0.4 . \stackrel{100}{ }$ This subdiffusive behavior (also called "anomalous diffusion") has been rationalized by several authors by means of simulations of water, both in inorganic $\underline{48,101}$ and organic confinement, $\underline{102}-\underline{105}$ with $\alpha$ exponents varying between $0.96 \underline{103}$ and $0.45 \pm 0.05 .101$ The proposed rationale is that the subdiffusive behavior is due to the heterogeneity of the surface and of the water-surface interaction. Nevertheless, this interpretation 

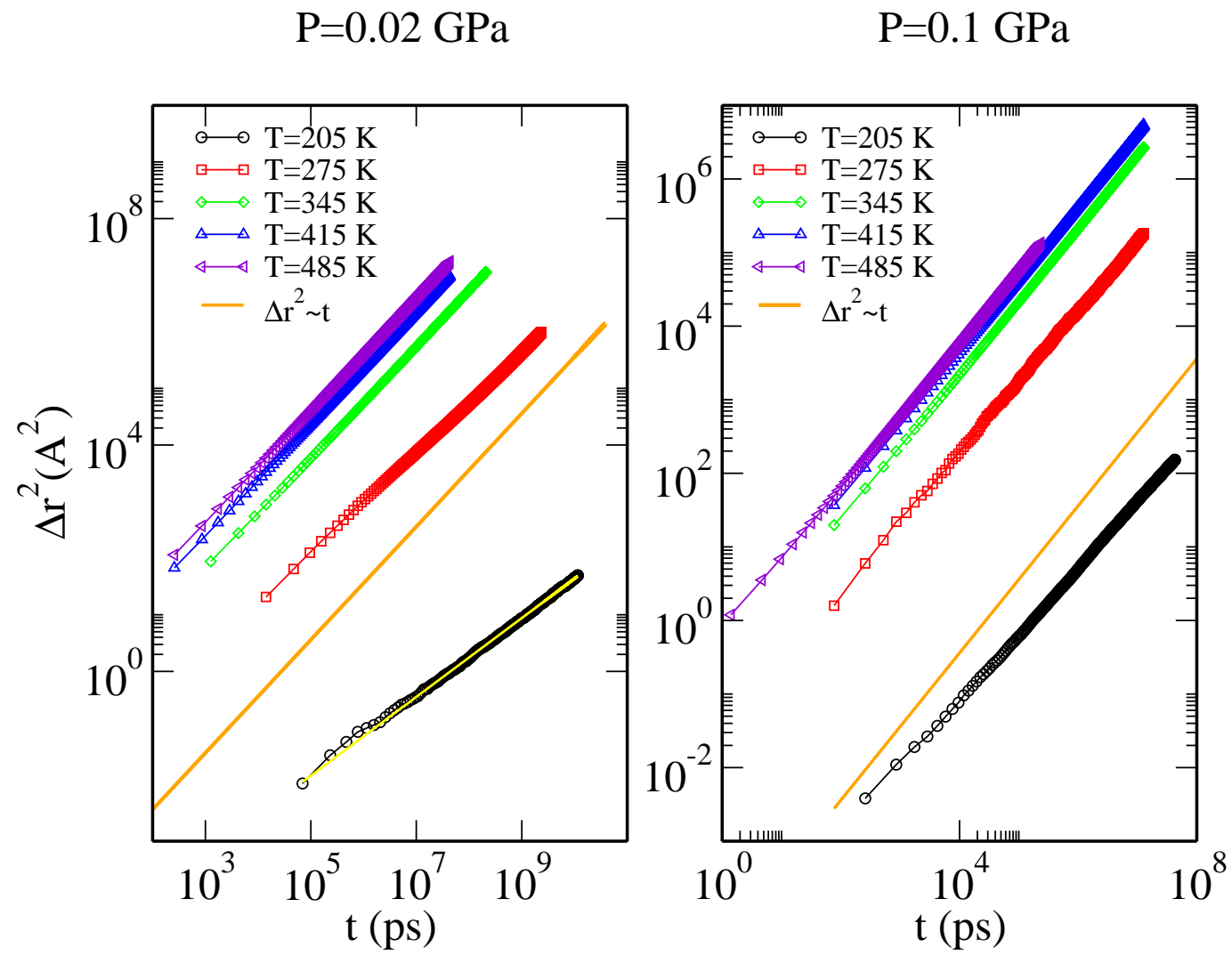

Figure 4: Mean square displacement $\Delta r^{2} \equiv\left\langle\left|\mathbf{r}_{i}\left(t+t_{0}\right)-\mathbf{r}_{i}(t)\right|^{2}\right\rangle$ as function of time $t$. In log-log plot, the diffusive regime $\Delta r^{2} \sim t$ has a slope 1 (parallel to the line without symbols). We calculate the diffusion coefficient $D_{\|}$from ?? within the diffusive regime. (Left panel) At $P=0.02 \mathrm{GPa}$, for $T \leq 275 \mathrm{~K}$ we find that the system does not reach the diffusive regime and the long-time behavior $\left(t>10^{6} \mathrm{ps}\right)$ is well described by the subdiffusive relation $\Delta r^{2} \sim t^{\alpha}$, with $\alpha=0.7$. (Right Panel) At higher pressure $P=0.1 \mathrm{GPa}$ the onset of subdiffusive regime occurs at lower $T$ with respect to the $P=0.02 \mathrm{GPa}$ case, for $T \leq 205 \mathrm{~K}$. Model parameters as in [figure][1][]1. 
does not apply to our case, where the surface is by definition homogeneous and flat and the watersurface interaction is only due to volume exclusion. In our case the subdiffusive dynamics is, instead, originated by the increasing correlation among the water molecules that will be discussed in the following subsections.

At higher pressure, $P=0.1 \mathrm{GPa}$, we find that the onset $T_{o}(P)$ of the subdiffusive regime occurs at about $T \leq 205 \mathrm{~K}$, i.e. at lower $T$ with respect to $P=0.02 \mathrm{GPa}([$ figure][4][]4). Therefore, within this range of $P$, the temperature $T_{o}(P)$ is correlated with $T_{\text {iso-D }}(P)$ of iso- $D_{\|}$lines, being $T_{\text {iso-D }}(P)-T_{o}(P) \simeq$ constant. This is no longer true at $P>0.22 \mathrm{GPa}([$ figure][1][]1) and can be understood in the framework of this model, because, as we will show in the next subsections, for $P>0.22 \mathrm{GPa}$ the number of HBs vanishes at low $T$ ([figure][6][]6).

\section{Density minima: relation with the cooperativity and the slow dynamics of the}

\section{HB network}
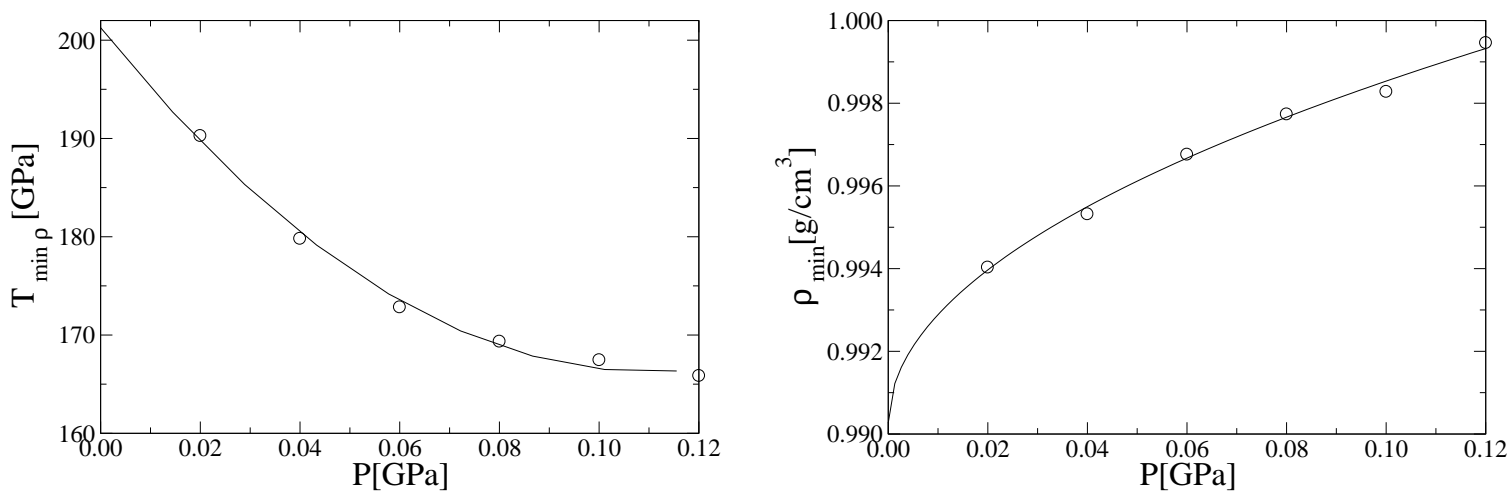

Figure 5: Density minima for the coarse-grained model of a water monolayer confined between hydrophobic walls. (Left panel) The locus of temperature $T_{\min \rho} \rho$ (circles) of minimum density as a function of pressures $P$ follows approximately a quadratic curve (continuous line) in the $P$ - $T$ plane, that extrapolates to about $201 \mathrm{~K}$ for atmospheric pressure. (Right panel) The density minima $\rho_{\min }$ (circles) display an approximate square-root dependence on pressure $P$, whose extrapolation for atmospheric pressure is about $0.99 \mathrm{~g} / \mathrm{cm}^{3}$. Model parameters as in [figure][1][]1.

Although for $T<T_{o}(P)$ our MC simulations become subdiffusive, we find that we can equilibrate the HBs dynamics within our simulation time for temperatures as low as $190 \mathrm{~K}$ at $0.02 \mathrm{GPa}$, 


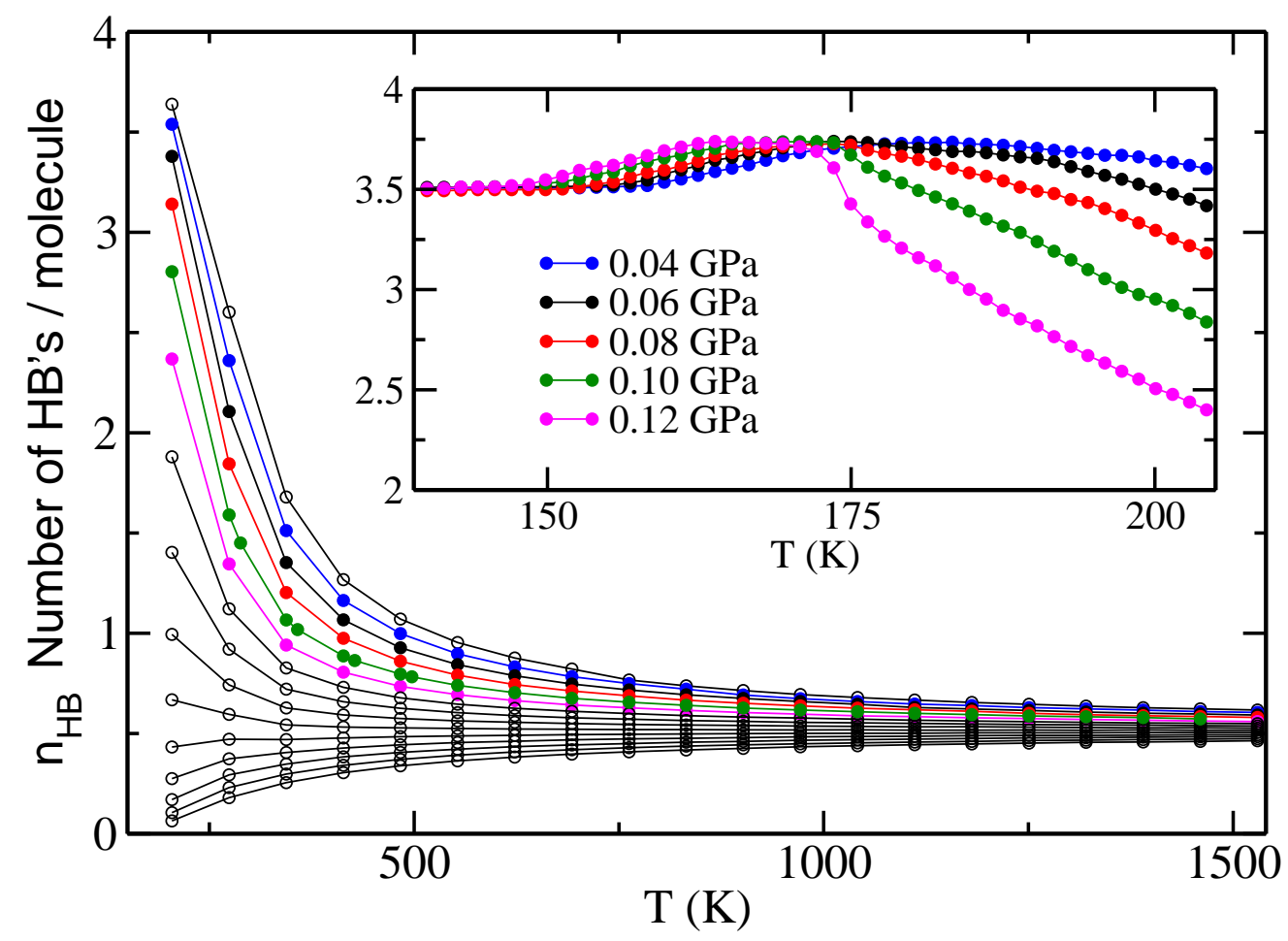

Figure 6: Number of hydrogen bonds $n_{H B}$ to which a molecule participate, as a function of temperature $T$ for pressures $P$ from (top to bottom) 0.02 to $0.3 \mathrm{GPa}$ in increments of $0.02 \mathrm{GPa}$. Inset: At low $T$ for $P$ from (top to bottom at $200 \mathrm{~K}$ ) 0.04 to $0.12 \mathrm{GPa}$ we find that $n_{H B}$ reaches a maximum value of $\sim 3.75$ at temperatures that coincide, within error bars, with those of the density minima $\rho_{\min }$ at the same pressure. Model parameters as in [figure][1][]1. 


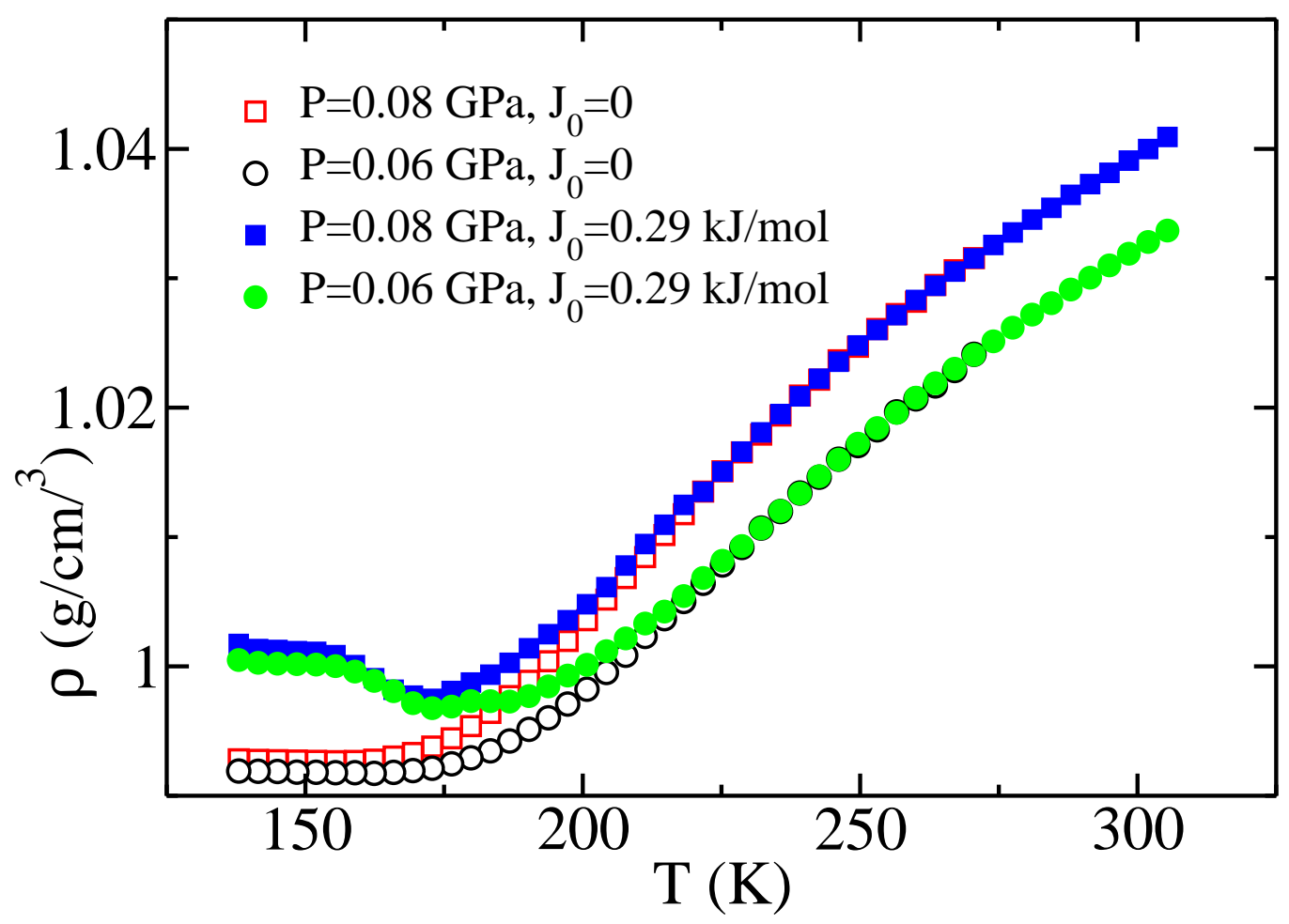

Figure 7: Comparison of the density behavior at low $T$ for the model with parameters as in [figure][1][]1 for $P=0.06 \mathrm{GPa}$ (full squares) and $P=0.08 \mathrm{GPa}$ (full circles), and with $J_{\sigma}=0$, and the other parameters unchanged, for the same pressures (open squares and open circles, respectively). We find that for $J_{\sigma}=0$ the density minima is not detectable within our resolution. 


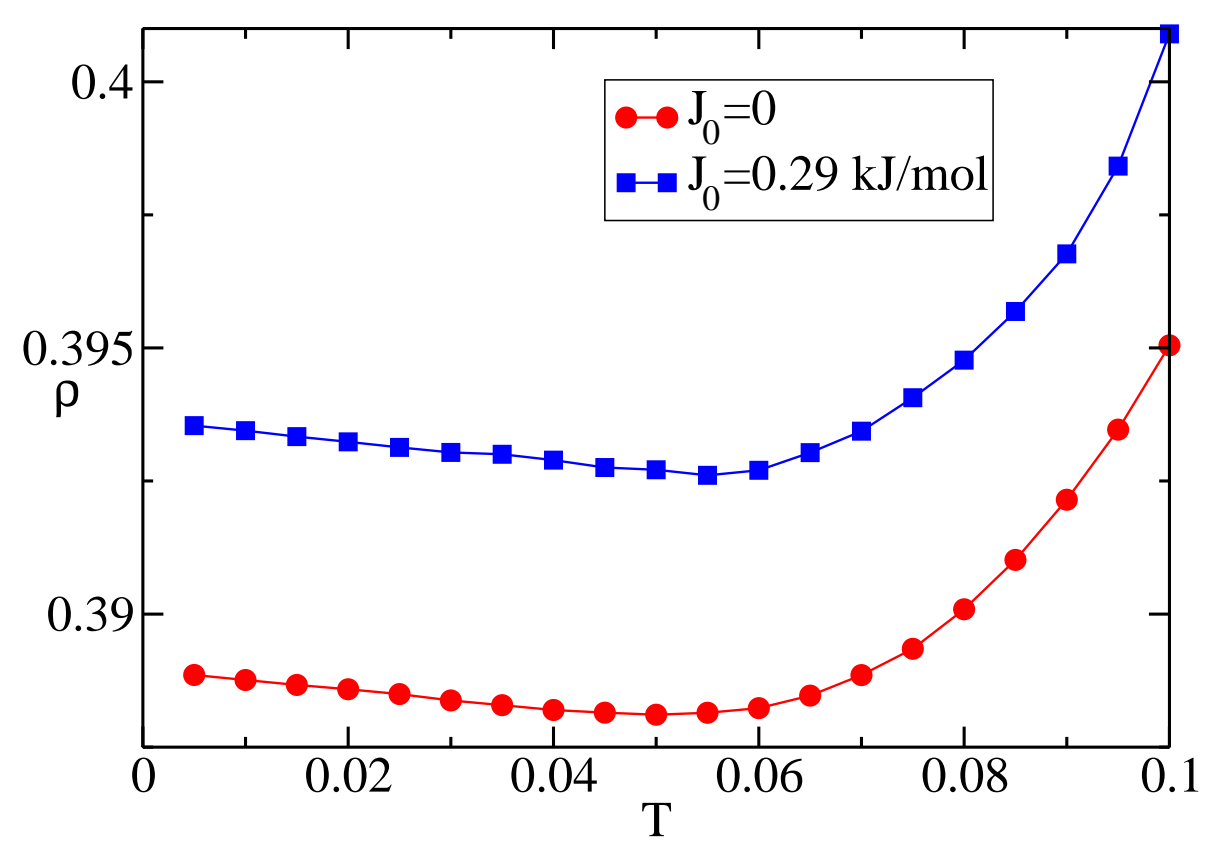

Figure 8: As in [figure][7][]7 but for $P=0.02 \mathrm{GPa}$, with parameters as in [figure][1][]1](squares) and with $J_{\sigma}=0$ (circles). The density minimum occurs also when $J_{\sigma}=0$.

or $163 \mathrm{~K}$ at $0.12 \mathrm{GPa}$. Specifically, we find that the relaxation time of the bonding indices $\sigma_{i j}$, related to the formation of the HBs, is of the order of $4 \mathrm{~ns}$ for these state points, while it exceeds our simulation time at lower $T$, e. g. at about $170 \mathrm{~K}$ for $P=0.02 \mathrm{GPa} . \underline{16}$

In the region of state points where we can equilibrate the system, but close to the lowest wellequilibrated temperature, we observe a minimum in density along isobars with $P \leq 0.12 \mathrm{GPa}$ ([figure][2][]2). This result resembles the experimental density minimum for water confined in a nanoporous silica matrix MCM-41 with a pore diameter of $1.4 \mathrm{~nm}$ found by Mallamace et al. $\underline{69}$

From our simulations for $P \geq 0.02 \mathrm{GPa}$, at atmospheric pressure we extract a density minimum of about $0.99 \mathrm{~g} / \mathrm{cm}^{3}$ at about $201 \mathrm{~K}$ ([figure][5][]5) not too far from the experimental value $0.940 \pm 0.003 \mathrm{~g} / \mathrm{cm}^{3}$ at about $203 \pm 5 \mathrm{~K}$ and atmospheric external pressure. $\frac{69}{}$ Although our simple quadratic extrapolation predicts a value for $\rho_{\min }$ that is larger than the experimental, our data give an extrapolated $T_{\min } \rho$ at atmospheric pressure consistent with the results of the experiments $\underline{69}$ and comparable to those from MD simulations of TIP5P-E water. $\underline{70}$

It must be noted, however, that the experimental results for confined water are controversial. $\underline{\underline{19}}$ Nevertheless, the controversy is mainly about the experimental measurement of the effect, and not 
about the effect, because it has been observed that the existence of a density minimum in water is a necessary consequence of the existence of the low- $P$ branch of the TMD line. $\underline{\underline{71}}$ In particular, it has been proposed that the locus of $\rho_{\min }$ corresponds to saturation, or the maximal ordering, of a network of water molecules with a random tetrahedral local arrangement. $\underline{\underline{71}}$

Our results, however, lead to a different explanation. We calculate the number of $\mathrm{HBs} n_{H B}$ in which a molecule participates, defined as $n_{H B} \equiv 2 N_{H B} / N$ from ?? in such a way as to have four as maximum value for each molecule of the coarse-grained monolayer. First, we observe that our simulation results for the monolayer are consistent with experimental data for bulk water, with $n_{H B} \simeq 0.45$ at $P=0.25 \mathrm{GPa}$ and $T \simeq 670 \mathrm{~K}$, and with $n_{H B} \simeq 2.2$ at the lowest $P$ at about ambient $T$, as reported in Ref. $\underline{\underline{81}}$ ([figure][6][]6).

Next, we find that for $P \leq 0.12$ GPa the quantity reaches a maximum value of $n_{H B} \simeq 3.75$ at temperatures that coincide, within error bars, with the temperatures $T_{\min } \rho$ of the density minima $\rho_{\min }$ at the same pressure, and $n_{H B}$ decreases to $\sim 3.5$ below $T_{\min } \rho$. Therefore, $N_{H B}$ decreases and for ?? the density increases.

To understand why $n_{H B}$ decreases below its maximum $n_{H B} \simeq 3.75$ at low $T$, we compare two cases corresponding to two different set of parameters of the model. The first as in [figure][1][]1. and the second, with $J_{\sigma}=0$ and the other parameters unchanged, corresponding to the LLCP and the SF scenario, respectively. $\underline{\underline{21}}$ By comparing the low- $T$ behavior of density for the two cases at intermediate $P$, we find that if $J_{\sigma}=0$, i.e. the hydrogen bond is not cooperative, then the density minima is undetectable within our resolution ([figure][7][]7].

However, at lower $P$ we find that both sets of parameters give a detectable density minimum ([figure][8][]8). Therefore, the cooperative term of the HB interaction is not essential for the occurrence of the density minima, but it emphasizes the minima at intermediate pressures. From this observation we understand that the explanation proposed by Poole at al. $\underline{\underline{ }}$ can be applied to the case with $J_{\sigma}=0$, in which the HB interaction does not include a cooperative (many body) term and the SF scenario is reproduced. In this case, the density increases for decreasing $T$ at very low $T$, when all the possible HBs have been formed, generating regions of mismatching tetrahedral local 
order. A decrease of $T$ induces a small reduction of free volume per molecule, and a consequent small increase of density.

Instead, when $J_{\sigma}>0$, the cooperative interaction in ?? induces the breaking of HBs for decreasing $T$, to allow the reorientation of a molecule and a better matching of local tetrahedral order at low $T$. As a consequence, the number $n_{H B}$ decreases from 3.75 to 3.5 inducing a large density increase. The high energy cost of this local rearrangement, i.e. the energy needed to break a HB, is at the origin of the high energy barrier for the process and the large increase of correlation time for the dynamics of the HB network in the vicinity of the locus of density minima.

\section{Relation of diffusion anomaly with different scenarios}

Several authors relate the diffusion anomaly in water to the presence of defects in the network of HBs. Here we show that the anomalous behavior of $D_{\|}$in the coarse-grained model is not related to the many-body component of the HB interaction and, therefore, to the possible occurrence of the LLCP. The anomaly is, instead, due to the anticorrelation between volume and entropy, or to volume and energy, rooted to HB formation.

We consider three different realizations of the coarse-grained model ([figure][9][]9). The first corresponds to the case presented in the previous section, with the parameters as in [figure][1][]1. For the second, we set $J_{\sigma}=0$, leaving the other parameters unchanged. This case reproduces the SF scenario, $\underline{\underline{7}}$ where density maxima occur and which has been shown to correspond to the vanishing-T limit of the LLCP. ${ }^{21}$ Our simulations show that the occurrence of the anomaly of $D_{\|}$is unaffected by this change of parameters $([$ figure $][9][] 9])$. Therefore, the absence of cooperativity in the HB dynamics is not relevant for the occurrence of both density maxima and diffusion anomaly.

This can be understood for the clear separation between the temperature range at which the anomaly of $D_{\|}$occurs and the temperature range at which water becomes subdiffusive. Only the latter regime corresponds to the temperature range for which the cooperativity has a strong influence on the dynamics, while it has no major dynamic effect at higher $T$.

Next, we set $v_{H B}=0$ and leave the other parameters as in [figure][1][1]. This case would 


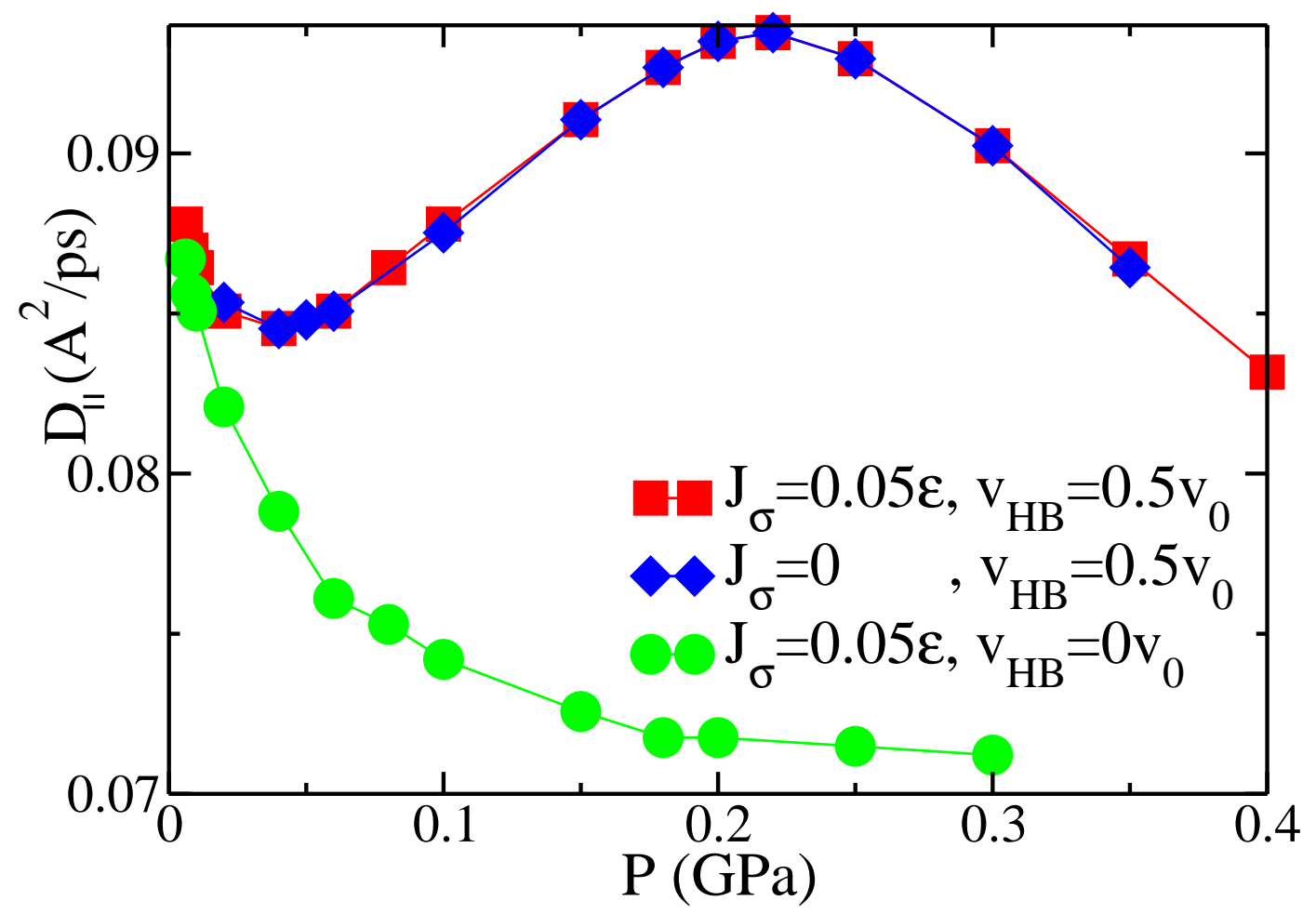

Figure 9: Effect of parameters $v_{\mathrm{HB}}$ and $J_{\sigma}$ on the diffusion anomaly. We diffusion minima and maxima for both $J_{\sigma}>0$ and $J_{\sigma}=0$. Instead, the non-monotonic behavior of $D_{\|}$disappears for $v_{\mathrm{HB}}=0$. 
correspond to cooperatively bonding liquid with no density anomaly, i.e. with no anticorrelation between volume and entropy or volume and energy. In this case the change in the behavior of $D_{\|}$ is striking $\left(\left[\right.\right.$ figure][9][]9]. The system has no diffusion anomaly, with $D_{\|}$that decreases monotonically for increasing $P$ as in normal liquids.

Therefore, this result clarifies that the anomalous volume behavior due to the HB formation is directly related to the anomalous diffusion behavior. This conclusion, and the previous observation that the many-body component of the $\mathrm{HB}$ interaction is not relevant in determining these anomalies, leads us to investigate how the volume available for diffusion, and the number of HBs are related to $D_{\|}$, as discussed elsewhere. $\underline{106}$

\section{Conclusions}

We study by Monte Carlo simulations a coarse-grained model for a water monolayer confined between hydrophobic walls. We consider a separation between walls about $h=0.5 \mathrm{~nm}$ that inhibits the formation of ice. $\underline{72}$

The model includes isotropic, directional (covalent) and many-body (cooperative) components of the HBs. Thanks to its coarse-graining, the model allows to study water in extreme conditions and, also, to check how each of the HB component affects its properties. Moreover, it makes possible to perform mean field calculations to compare with simulations results.

We find gas and liquid phases, separated by a boundary line of first-order phase transitions ending in a critical point occurring at higher pressure and temperature, consistent with other models for hydrophobically confined water. $\underline{.6}$ We study the diffusion constant parallel to the walls $D_{\|}$ and find that it displays a line of maxima and a line of minima at constant $T$, as seen in similar confinement for other models. $\underline{\underline{49}}$ Our analysis allows us to conclude that the anomalous $D_{\|}$is a consequence of the anomalous volume behavior due to HB formation. In particular, the positive correlation between entropy and density, or energy and density, due to hydrogen bonding is the key element for the diffusion anomaly. It is worth reminding here that a similar result has been found 
also for potentials with isotropic interactions and water-like anomalies $\underline{10,36,107}$ when the isothermal density dependence of the excess entropy, which is related to the total isothermal entropy by a linear function of the logarithm of the density, is considered.

The difference with the present analysis is, nevertheless, threefold. First, for these isotropic potentials the HDL phase has less entropy than the LDL phase, implying a positive slope in the $P-T$ plane for the liquid-liquid phase coexistence line as a consequence of the Clausius-Clapeyron equation. Instead, for water and the present model the HDL phase has more entropy than the LDL phase, hence the liquid-liquid phase transition has a negative slope in the $P-T$ plane.

Second, here we show that by setting the parameter that controls the increase of volume for HB formation, hence the positive correlation of density with entropy and energy, the anomalous diffusion behavior vanishes. Instead, for the isotropic potentials the vanishing of the anomalous diffusion behavior is controlled by the softness of the soft-core repulsion of the potential. $\frac{10,11}{\mathrm{~A}}$ direct relation between these two results is not straightforward and could be interesting to investigate.

Third, the present result does not exclude that the key element for diffusion anomaly is the positive correlation of density and energy, instead of entropy. While in water and the present model the LDL phase has lower energy and entropy than the HDL, in the isotropic potentials with water-like anomalies the LDL phase has lower energy but higher entropy than the HDL. These considerations support the idea that the mechanism of anomalies in isotropic potentials is different from that of water. $\underline{9}$

Interestingly, here we also observe that the lines of constant $D_{\|}$resemble the melting line of bulk water. At low temperatures, we find the locus of density maxima, which marks another wellknown water anomaly. We discuss how this locus is related to the locus of expansivity minima.

At lower $T$, we find subdiffusive behavior $\Delta r^{2} \sim t^{\alpha}$, as seen in experiments of hydration wa-

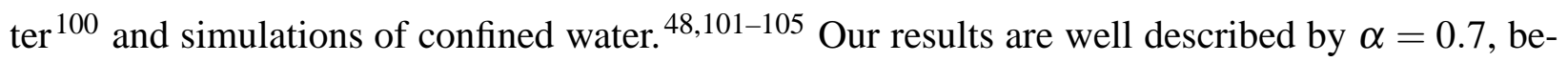
tween $0.96^{103}$ and $0.45 \pm 0.05 \frac{101}{10}$ of previous calculations. Previous works proposed that subdiffusion is a consequence of the heterogeneities in water-interface interaction. Here this rationale does 
not apply and we relate the subdiffusion to the increase of correlation among water molecules at low $T$ due to the full development of the HB network.

By further decreasing $T$, we find density minima, as seen in experiments $\frac{69}{}$ and MD simulations. $\stackrel{70.71}{ }$ These minima occur within the subdiffusive part of the phase diagram, therefore where translational motion is strongly hampered and glassy behavior is incipient. Nevertheless, the HB network within this region is still dynamically evolving, with increasing correlation time. ${ }^{16}$ In particular, the HB correlation time is about $4 \mathrm{~ns}$ for the majority of the subdiffusive region and increases, exceeding our simulation times of the order of $15 \mathrm{~ms}$, only at about $P \leq 0.02 \mathrm{GPa}$ and $T \leq 170 \mathrm{~K}$

Previous works related the density minima to the saturation of a network of molecules with a random tetrahedral local arrangement. $\stackrel{71}{ }$ However, this rationale apply to our model only for the case in which the cooperative component of the hydrogen bonds is zero. When the cooperative component is larger than zero, as expected in real water, $\stackrel{25}{\underline{2}}$ our calculations show that the minima are due to a reduction of the number of HBs, as a consequence of the reorientation of molecules for a better matching of local order. The high energy cost of this rearrangement is the cause of the large slowing down of the HB dynamics near the state points where the density minima occur.

\section{Acknowledgement}

FDLS acknowledges support from projects nos. FIS2009-08451 (MICINN) and P07-FQM02725 (Junta de Andalucía). GF acknowledges support from MICINN project n. FIS2009-10210 (cofinanced FEDER).

\section{References}

(1) Debenedetti, P. G.; Stanley, H. E. Physics Today 2003, 56, 40-46.

(2) Franzese, G.; Stanley, H. E. In Water and Life: The Unique Properties of $\mathrm{H}_{2} \mathrm{O}$; Lynden- 
Bell, R. M., Conway Morris, S., Barrow, J. D., Finney, J. L., Harper, C., Eds.; CRC Press, 2010; Chapter 7

(3) At least 67 anomalies are listed in the well documented website http://www.lsbu.ac.uk/water/anmlies.html.

(4) Debenedetti, P. G. Metastable Liquids. Concepts and Principles; Princeton University Press: Princeton, NJ, 1996.

(5) Speedy, R. J. The Journal of Physical Chemistry 1982, 86, 3002-3005.

(6) Poole, P.; Sciortino, F.; Essmann, U.; Stanley, H. Nature 1992, 360, 324-328.

(7) Sastry, S.; Debenedetti, P. G.; Sciortino, F.; Stanley, H. E. Physical Review E 1996, 53, $6144-6154$.

(8) Angell, C. A. Science 2008, 319, 582-587.

(9) Franzese, G.; Malescio, G.; Skibinsky, A.; Buldyrev, S. V.; Stanley, H. E. Nature 2001, 409, 692-695.

(10) Vilaseca, P.; Franzese, G. The Journal of Chemical Physics 2010, 133, 084507.

(11) Vilaseca, P.; Franzese, G. Journal of Non-Crystalline Solids 2011, 357, 419 - 426.

(12) Abascal, J. L. F.; Vega, C. The Journal of Chemical Physics 2011, 134, 186101.

(13) Abascal, J. L. F.; Vega, C. The Journal of Chemical Physics 2010, 133, 234502.

(14) Liu, Y.; Panagiotopoulos, A. Z.; Debenedetti, P. G. The Journal of Chemical Physics 2009, $131,104508$.

(15) Franzese, G.; Stanley, H. E. Journal of Physics-Condensed Matter 2002, 14, 2201-2209. 
(16) Franzese, G.; de los Santos, F. J. Phys.: Condens. Matter 2009, 21, 504107.

(17) Bellissent-Funel, M.-C.; Chen, S. H.; Zanotti, J.-M. Physical Review E 1995, 51, 45584569.

(18) Zhang, Y.; Liu, K. H.; Lagi, M.; Liu, D.; Littrell, K. C.; Mou, C. Y.; Chen, S. H. Journal of Physical Chemistry B 2009, 113, 5007-5010.

(19) Mancinelli, R.; Bruni, F.; Ricci, M. A. The Journal of Physical Chemistry Letters 2010, 1, $1277-1282$.

(20) Strekalova, E. G.; Mazza, M. G.; Stanley, H. E.; Franzese, G. Physical Review Letters 2011, $106,145701$.

(21) Stokely, K.; Mazza, M. G.; Stanley, H. E.; Franzese, G. Proceedings of the National Academy of Sciences of the United States of America 2010, 107, 1301-1306.

(22) Jonas, J.; DeFries, T.; Wilbur, D. J. The Journal of Chemical Physics 1976, 65, 582-588.

(23) Prielmeier, F. X.; Lang, E. W.; Speedy, R. J.; Lüdemann, H. D. Physical Review Letters 1987, 59, 1128-1131.

(24) Prielmeier, F. X.; Lang, E. W.; Speedy, R. J.; Luedemann, H. D. Berichte der Bunsengesellschaft für Physikalische Chemie 1988, 92, 1111-1117.

(25) Ludwig, R. Angewandte Chemie International Edition 2001, 40, 1808-1827.

(26) Reddy, M. R.; Berkowitz, M. The Journal of Chemical Physics 1987, 87, 6682-6686.

(27) Gallo, P.; Sciortino, F.; Tartaglia, P.; Chen, S. H. Physical Review Letters 1996, 76, 27302733.

(28) Starr, F. W.; Harrington, S.; Sciortino, F.; Stanley, H. E. Physical Review Letters 1999, 82, 3629-3632. 
(29) Starr, F. W.; Sciortino, F.; Stanley, H. E. Physical Review E 1999, 60, 6757-6768.

(30) Netz, P. A.; Starr, F. W.; Stanley, H. E.; Barbosa, M. C. The Journal of Chemical Physics 2001, 115, 344-348.

(31) Girardi, M.; Szortyka, M.; Barbosa, M. C. Physica A-Statistical Mechanics And Its Applications 2007, 386, 692-697.

(32) Szortyka, M. M.; Barbosa, M. C. Physica A: Statistical Mechanics and its Applications 2007, 380, 27-35.

(33) Szortyka, M. M.; Fiore, C. E.; Henriques, V. B.; Barbosa, M. C. The Journal of Chemical Physics 2010, 133, 104904.

(34) Scala, A.; Starr, F. W.; La Nave, E.; Sciortino, F.; Stanley, H. E. Nature 2000, 406, 166-169.

(35) Errington, J. R.; Debenedetti, P. G. Nature 2001, 409, 318-321.

(36) Errington, J. R.; Truskett, T. M.; Mittal, J. The Journal of Chemical Physics 2006, 125, 244502.

(37) Fernández-Serra, M. V.; Artacho, E. The Journal of Chemical Physics 2004, 121, 1113611144.

(38) Kamitakahara, W. A.; Wada, N. Physical Review E 2008, 77, 041503-10.

(39) Major, R. C.; Houston, J. E.; McGrath, M. J.; Siepmann, J. I.; Zhu, X. Y. Physical Review Letters 2006, 96, 177803.

(40) Takahara, S.; Nakano, M.; Kittaka, S.; Kuroda, Y.; Mori, T.; Hamano, H.; Yamaguchi, T. The Journal of Physical Chemistry B 1999, 103, 5814-5819.

(41) Faraone, A.; Liu, L.; Mou, C.-Y.; Shih, P.-C.; Copley, J. R. D.; Chen, S.-H. The Journal of Chemical Physics 2003, 119, 3963-3971. 
(42) Mallamace, F.; Broccio, M.; Corsaro, C.; Faraone, A.; Wanderlingh, U.; Liu, L.; Mou, C.-Y.; Chen, S. H. The Journal of Chemical Physics 2006, 124, 161102.

(43) Chen, S.-H.; Mallamace, F.; Mou, C.-Y.; Broccio, M.; Corsaro, C.; Faraone, A.; Liu, L. Proceedings of the National Academy of Sciences of the United States of America 2006, $103,12974-12978$.

(44) Naguib, N.; Ye, H.; Gogotsi, Y.; Yazicioglu, A. G.; Megaridis, C. M.; Yoshimura, M. Nano Letters 2004, 4, 2237-2243.

(45) Holt, J. K.; Park, H. G.; Wang, Y.; Stadermann, M.; Artyukhin, A. B.; Grigoropoulos, C. P.; Noy, A.; Bakajin, O. Science 2006, 312, 1034-1037.

(46) Majumder, M.; Chopra, N.; Andrews, R.; Hinds, B. J. Nature 2005, 438, 44-44.

(47) Romero-Vargas Castrillón, S.; Giovambattista, N.; Aksay, I. A.; Debenedetti, P. G. The Journal of Physical Chemistry B 2009, 113, 7973-7976.

(48) Gallo, P.; Rovere, M.; Chen, S.-H. Journal of Physics: Condensed Matter 2010, 22, 284102.

(49) Kumar, P.; Buldyrev, S. V.; Starr, F. W.; Giovambattista, N.; Stanley, H. E. Physical Review E 2005, 72, 051503.

(50) Han, S.; Kumar, P.; Stanley, H. E. Physical Review E 2008, 77, 030201.

(51) Choudhury, N.; Pettitt, B. M. The Journal of Physical Chemistry B 2005, 109, 6422-6429.

(52) Cicero, G.; Grossman, J. C.; Schwegler, E.; Gygi, F.; Galli, G. Journal of the American Chemical Society 2008, 130, 1871-1878.

(53) Martí, J.; Gordillo, M. C. Physical Review E 2001, 64, 021504.

(54) Mashl, R. J.; Joseph, S.; Aluru, N. R.; Jakobsson, E. Nano Letters 2003, 3, 589-592.

(55) Liu, Y.; Wang, Q.; Wu, T.; Zhang, L. The Journal of Chemical Physics 2005, 123, 234701-7. 
(56) Mazza, M. G.; Stokely, K.; Strekalova, E. G.; Stanley, H. E.; Franzese, G. Computer Physics Communications 2009, 180, 497-502.

(57) Cataudella, V.; Franzese, G.; Nicodemi, M.; Scala, A.; Coniglio, A. Physical Review E 1996, 54, 175-189.

(58) Franzese, G. Journal of Physics A-Mathematical and General 1996, 29, 7367-7375.

(59) Franzese, G.; Coniglio, A. Physical Review E 1999, 59, 6409-6412.

(60) Franzese, G.; Bianco, V.; Iskrov, S. Food Biophysics 2011, 6, 186-198, 10.1007/s11483010-9198-4.

(61) Kumar, P.; Franzese, G.; Stanley, H. E. Physical Review Letters 2008, 100, 105701.

(62) Kumar, P.; Franzese, G.; Stanley, H. E. Journal of Physics: Condensed Matter 2008, 20, 244114.

(63) Franzese, G.; Coniglio, A. Physical Review E 1998, 58, 2753-2759.

(64) Fierro, A.; Franzese, G.; de Candia, A.; Coniglio, A. Physical Review E 1999, 59, 60-66.

(65) Gotze, W.; Sjogren, L. Reports on Progress in Physics 1992, 55, 241-376.

(66) Gleim, T.; Kob, W.; Binder, K. Physical Review Letters 1998, 81, 4404.

(67) Saw, S.; Ellegaard, N. L.; Kob, W.; Sastry, S. The Journal of Chemical Physics 2011, 134, 164506.

(68) Mazza, M. G.; Stokely, K.; Pagnotta, S. E.; Bruni, F.; Stanley, H. E.; Franzese, G. Two dynamic crossovers in protein hydration water and their thermodynamic interpretation. 2009; http://arxiv.org/abs/0907.1810,

(69) Mallamace, F.; Branca, C.; Broccio, M.; Corsaro, C.; Mou, C.-Y.; Chen, S.-H. Proceedings of the National Academy of Sciences of the United States of America 2007, 104, 1838718391. 
(70) Paschek, D. Physical Review Letters 2005, 94, 217802.

(71) Poole, P. H.; Saika-Voivod, I.; Sciortino, F. Journal of Physics: Condensed Matter 2005, 17, L431-L437.

(72) Zangi, R.; Mark, A. E. Physical Review Letters 2003, 91, 025502.

(73) Narten, A. H.; Danford, M. D.; Levy, H. A. Discussions of the Faraday Society 1967, 43, 97.

(74) Soper, A.; Ricci, M. Physical Review Letters 2000, 84, 2881-2884.

(75) Henry, M. Chemphyschem 2002, 3, 561-9.

(76) Isaacs, E. D.; Shukla, A.; Platzman, P. M.; Hamann, D. R.; Barbiellini, B.; Tulk, C. A. Journal of Physics and Chemistry of Solids 2000, 61, 403 - 406.

(77) Pendas, A. M.; Blanco, M. A.; Francisco, E. The Journal of Chemical Physics 2006, 125, 184112.

(78) Suresh, S. J.; Naik, V. M. The Journal of Chemical Physics 2000, 113, 9727-9732.

(79) Soper, A. K. Chemical Physics 2000, 258, 121-137.

(80) Soper, A. K. Molecular Physics 2008, 106, 2053 - 2076.

(81) Bernabei, M.; Botti, A.; Bruni, F.; Ricci, M. A.; Soper, A. K. Physical Review E 2008, 78, 021505 .

(82) Ricci, M. A.; Bruni, F.; Giuliani, A. Faraday Discuss. 2009, 141, 347-358.

(83) Eisenberg, D.; Kauzmann, W. The Structure and Properties of Water; Oxford University Press, 1969; p 139.

(84) Heggie, M. I.; Latham, C. D.; Maynard, S. C. P.; Jones, R. Chemical Physics Letters 1996, $249,485-490$. 
(85) Franzese, G.; Yamada, M.; Stanley, H. E. AIP Conference Proceedings 2000, 519, 281 287.

(86) Franzese, G.; Stanley, H. E. Physica A-Statistical Mechanics And Its Applications 2002, 314, 508-513.

(87) Franzese, G.; Marqués, M. I.; Stanley, H. E. Physical Review E 2003, 67, 011103.

(88) Franzese, G.; Stanley, H. E. In Complexity, Metastability And Nonextensivity; Beck, C., Benedek, G., Rapisarda, A., Tsallis, C., Eds.; Science And Culture Series: Physics; World Scientific, 2005; Vol. 26; Chapter The metastable liquid-liquid phase transition: from water to colloids and liquid metals, pp 210-214.

(89) Franzese, G.; Stanley, H. E. Journal of Physics-Condensed Matter 2007, 19, 205126.

(90) Kumar, P.; Franzese, G.; Buldyrev, S. V.; Stanley, H. E. In Aspects of Physical Biology; Franzese, G., Rubí, M., Eds.; Lecture Notes in Physics; Springer Berlin / Heidelberg, 2008; Vol. 752; Chapter Dynamics of Water at Low Temperatures and Implications for Biomolecules, pp 3-22.

(91) Franzese, G.; Stokely, K.; Chu, X. Q.; Kumar, P.; Mazza, M. G.; Chen, S. H.; Stanley, H. E. Journal of Physics-Condensed Matter 2008, 20, 494210.

(92) Mazza, M. G.; Stokely, K.; Stanley, H. E.; Franzese, G. Anomalous specific heat of supercooled water. 2008; http://arxiv.org/abs/arXiv:0807.4267.

(93) de los Santos, F.; Franzese, G. In Modeling And Simulation Of New Materials: Proceedings Of Modeling And Simulation Of New Materials: Tenth Granada Lectures; Marro, J., Garrido, P. L., Hurtado, P. I., Eds.; AIP Conf. Proc.; AIP, 2009; Vol. 1901; Chapter Influence of intramolecular couplings in a model for hydrogen-bonded liquids, pp 185-197.

(94) Franzese, G.; Hernando-Martínez, A.; Kumar, P.; Mazza, M. G.; Stokely, K.; 
Strekalova, E. G.; de los Santos, F.; Stanley, H. E. Journal of Physics: Condensed Matter 2010, 22, 284103.

(95) Stokely, K.; Mazza, M. G.; Stanley, H. E.; Franzese, G. In Metastable Systems under Pressure; Rzoska, S. J., Drozd-Rzoska, A., Mazur, V. A., Eds.; NATO Science for Peace and Security Series A: Chemistry and Biology; Springer, 2010; Chapter Metastable Water Under Pressure, pp 197-216.

(96) Gallo, P.; Rovere, M. Physical Review E 2007, 76, 061202-7.

(97) Sorensen, C. M. The Journal of Chemical Physics 1983, 79, 1455-1461.

(98) Hare, D. E.; Sorensen, C. M. The Journal of Chemical Physics 1986, 84, 5085-5089.

(99) Fuentevilla, D. A.; Anisimov, M. A. Physical Review Letters 2006, 97, 195702.

(100) Settles, M.; Doster, W. Faraday Discuss. 1996, 103, 269-279.

(101) Gallo, P.; Rovere, M. Journal of Physics: Condensed Matter 2003, 15, 7625-7633.

(102) Bizzarri, A. R.; Rocchi, C.; Cannistraro, S. Chemical Physics Letters 1996, 263, 559-566.

(103) Bizzarri, A. R.; Cannistraro, S. Physical Review E 1996, 53, R3040-R3043.

(104) Rocchi, C.; Bizzarri, A. R.; Cannistraro, S. Physical Review E 1998, 57, 3315-3325.

(105) Oleinikova, A.; Smolin, N.; Brovchenko, I. Biophysical Journal 2007, 93, 2986-3000.

(106) de los Santos, F.; Franzese, G. in preparation 2011,

(107) de Oliveira, A. B.; Franzese, G.; Netz, P. A.; Barbosa, M. C. The Journal of Chemical Physics 2008, 128, 064901 